\title{
A QUANTITATIVE ASSESSMENT MODEL FOR ICT SYSTEM SOLUTION SELECTION WITH BAYESIAN NETWORK FOR ENTERPRISE ARCHITECTURE DECISIONS
}

\author{
DOI: 10.17261/Pressacademia.2018.897 \\ PAP- V.7-2018(50)-p.277-280
}

Pinar Yildiran ${ }^{1}$, Huseyin Selcuk Kilic ${ }^{2}$, Bahar Sennaroglu ${ }^{3}$

${ }^{1}$ Marmara University, Goztepe Yerleskesi 34722 Kadıköy, Istanbul, Turkey. pinaryildiran@marun.edu.tr, ORCID: 0000-0001-7699-6447

${ }^{2}$ Marmara University, Goztepe Yerleskesi 34722 Kadıköy, Istanbul, Turkey. huseyin.kilic@marmara.edu.tr, ORCID: 0000-0003-3356-0162

${ }^{3}$ Marmara University, Goztepe Yerleskesi 34722 Kadıköy, Istanbul, Turkey. bsennar@marmara.edu.tr, ORCID: 0000-0002-6809-634X

To cite this document

Yildiran, P., Kilic, H. S., Sennaroglu, B. (2018). A quantitative assessment model for IT system solution selection with Bayesian network for enterprise architecture decisions. PressAcademia Procedia (PAP), V.7, p.277-280.

Permemant link to this document: http://doi.org/10.17261/Pressacademia.2018.897

Copyright: Published by PressAcademia and limited licenced re-use rights only.

\section{ABSTRACT}

Purpose- This research proposes a quantitative assessment model for ICT System Solution Selection

Methodology- For large Enterprise Organizations, ICT Projects may have two alternative solutions such as centralized or distributed ICT Systems. For evaluating these options with Enteprise Architecture principles and frameworks and also bringing expert knowledge in decision support, "Quantitative Assessment Model Survey for IT Solution Selection" study is conducted among ICT professionals of various industrial sectors. To build the model for centralized or distributed ICT system solutions, the influential relations between the surveyed EA components and principles are identified with Structural Learning of Bayesian Network to make the relations of the important EA elements visible and quantifiable.

Findings- Using data from our survey, adaptable quantitative model which enables a Bayesian network across organized EA components is developed.

Conclusion- The study shows relationships between EA components with experts' feedbacks which may help practitioners and decision makers to reach a common model for ICT solution evaluation.

Keywords: Structural learning, Bayesian network, enterprise engineering, enterprise architecture, decision making. JEL Codes: $\mathrm{C} 44, \mathrm{C} 11, \mathrm{D} 81$

\section{INTRODUCTION}

We live in a constantly and rapidly changing environment due to new developments and innovations in science and technology in line with human evolution. This brings complexity and challenge to businesses that need instruments and methods to continue and to transform their operations consequently. With the new technologies the alignment and integration of business and ICT is a mandatory action for every business in digital era. Business capabilities and processes in Enterprise organizations are strongly tight with Information and Communication Systems' (ICT) capabilities on top of Enterprise Engineering. Enterprise Architecture (EA) is a promising and comprehensive approach to model the current and the future state of Enterprises. EA helps to analyze and to structure of ICT systems of the Enterprise Organizations. With the complexity concept in Enterprise organizations the purpose is to handle the various aspects and interdependencies between these aspects of the Enterprises as a whole. In general most of the time ICT projects are encompassed with significant risks. Due to the high level uncertainty and dynamic changes of competitive environment and unpredictable nature of human factors, the quantitative and rational EA decisions with the help of EA frameworks play an important deterministic role in ICT projects. The identification and evaluation of ICT projects to be invested is a hard problem that Enterprise Architecture addresses. However it is obvious that especially human factor plays very important role for key decision making process to have a proper EA for ICT projects in Enterprise IT organizations. For selection and/or founding of proper methods, processes and tools in line with EA frameworks by the identified key factors and key principles, a practical and quantitative assessment model was proposed for EA decision making, possibly in other words Enterprise Engineering decision making.

In literature review, although the various benefits of EA, the value proposition of EA was not put in practice especially for proactive decision making examples. With this research, a quantitative assessment model was derived for ICT System Solution Selection in ICT projects. For especially large Enterprise Organizations, ICT Projects may have two alternative solutions such as centralized or distributed ICT Systems. For evaluating these options in line with EA principles and frameworks and also bringing vast expert knowledge in decision 
support, the "Quantitative Assessment Model Survey for IT Solution Selection" study was conducted among ICT professionals of various industrial sectors. In order to build the assessment model for centralized or distributed ICT system solutions, the influential relations between the surveyed EA components and principles were identified with the use of Structural Learning of Bayesian Network to make the relations of the important EA elements visible and quantifiable. This modelling provides an interesting point of view for the analysis and design of Enterprise as a complex system in Enterprise Engineering. The proposed model formalizes explicitly the combining of these EA principles and factors to support the decision process. At the same time, the generic statistical approaches guide to enable the examination of the preferability of the decision alternatives either centralized or distributed ICT System Solution contexts. Within this research the survey contributes to a better understanding of Enterprise Architecture components for ICT project solution selection. Also the expert data elicitation explicitly serves for quantifying uncertainty to model complex relationships among them with the methodology of defining the causal relations between EA components as variables and learning from data to update previous beliefs and probabilities in applying the model to a project to select the proper IT solution whether centralized or distributed. For Enterprise Architecture Frameworks TOGAF has been selected for identifying the EA components. TOGAF is a framework with a comprehensive method and related tools for building and enhancing an enterprise architecture. TOGAF (The Open Group Architecture Framework) is a prominent standard for the development and deployment of modern IT systems in Enterprise organizations (Dietz \& Hoogervorst, 2011).

This research does not define a new decision making process but enables a new way of experimentation and practical system decision process with the combination of Bayesian Methodology on a mathematical basis of calculations and with the Enterprise Architecture Framework known as TOGAF in order to improve decision deficiencies for high level management. The significance of choosing Bayesian analysis for this research is that Bayesian methods can handle the complexity and well suited for decision-making under uncertainty. The paper shows how the optimal centralized or distributed ICT system solution selection model can be obtained efficiently.

The paper is organized as follows. "Section 2" presents background research and literature review that builds the foundations for all following sections in Enterprise Architecture and Enterprise Engineering. "Section 3" gives the basis of proposed ICT Solution Selection Model with the computational methodologies and related explanations. A numerical sample illustration of the model is given in "Section 4 in order to provide preliminary application of our work and finally, in "Section 5" discussion of ideas and contributions behind this work is presented with a conclusion.

\section{LITERATURE REVIEW AND BACKGROUND RESEARCH}

This section provides the background of our enterprise operational decision-making model and analysis of related concepts for better understanding. It reviews briefly the previous research in this field and related literature and it gives an overview of the major concepts and constructs related with developing our proposed model.

Over the last decades, there has been a particular alignment perspective between ICT Solutions and Business Processes with architecturedriven ICT systems. ICT takes an enabler role for business support with increasing flexibility and adaptability of the business activities in Enterprise Organizations. 'Enterprise' is a term to identify a company, organization, business or governmental institution (Dietz et al., 2009) and encompassing all of its information and technology services, processes and infrastructure. TOGAF defines "enterprise' 'as any collection of organizations that has a common set of goals. An enterprise delivers products and services to accomplish enterprise results and goals (Presley et al., 1996). Enterprise has processes, people, information and technology for realizing its own core componets as products and services. Within the design and change view Enterprise Architecture shows the relationship among Enterprise's core components (Yildiran et al., 2018). Enterprises are complex and dynamic systems (Kosanke et al., 1999) and change of Enterprise core components is a continuous process as a natural cycle. For business and ICT alignment in all aspects of Enterprise systems, Enterprise Architecture supports the decision making and realization of Enterprise transformation from "current structure" to "to be structure" of Enterprise organizations by using generic EA frameworks, methods and language. The technical architecture drives standards, techniques, and policy-based aspects of the application architecture (Perks and Beveridge 2007). An Enterprise Architecture Framework actually defines the interactions and relationships between the enterprise's elements (people, processes, technology etc.) EA frameworks contain "toolboxes" such as sets of model approaches, processes, and taxonomies in their reference architecture to deliver business outcomes.

There are many architectural frameworks in use today that differs according to specific needs/concerns of Enterprise organizations. There is no single "best" enterprise architecture framework applicable to all enterprises and for all their applications. Each framework has its strengths and weaknesses so enterprises may choose a blended approach by applying best practices or using different models, representations for choosing the right framework (Sessions, 2007). There is common agreement on to use and architectural framework views of enterprise architecture. These frameworks provide ontology and different abstraction levels to map enterprise information for helping the Enterprise Architects to communicate the architectural facts and relationships of Enterprise (Greefhorst and Proper, 2011). Famous EA Frameworks can be listed as The Zachman Framework, TOGAF, CIMOSA, DODAF, MODAF and FEAF'. Although many architecture reference frameworks are defined, the systematic literature review indicates that the researchers mostly focus on TOGAF and only few Enterprise Architecture Reference models are used widely.

Enterprise Architecture and Enterprise Engineering compose of many concepts and their relationships. The literature review shows that there is a lack of research on Enterprise Engineering and Enterprise Architecture as a whole. While the most important issue about Enterprise Engineering and Enterprise Architecture is the design decisions of Enterprise, there is lack of decision support in use particularly in IT organizations (Yildiran et al., 2018). Most studies on theoretical and conceptual models are based on qualitative approaches and few studies use quantitative methods, which is considered as contribution of this study to the field. 


\section{THE PROPOSED METHODOLOGY}

In this section the main concepts of approach and methodology used to achieve the research goal and the specific objectives were explained briefly according to the research methodology structure.

Probabilistic networks and cognitive maps play an important role in the proposed methodology, probabilistic networks are so popular for reasoning under uncertainty, for diagnosis, prediction, decision making, classification, and data mining. Whereas, a cognitive map or known as causal map (CM) is the representation of thinking on a problem related to an individual's cognition process (Harary et al., 1965; Harary, 1972). Cognitive maps in general are subjective methods that gather information from experts by using their knowledge and experiences and usually derived through interviews for the necessity of taking into account numerous aspects and interdependencies (Eden, 2004). Experts express themselves in qualitative way. The major concern in $\mathrm{CM}$ is to define the magnitude of the effect to express in numbers and $\mathrm{CM}$ can be analyzed quantitatively as with matrix algebra and network analytic methods and qualitatively. Causal Maps are useful for building Bayesian Networks. Bayesian (or belief) networks show graphically probabilistic relationship between a set of variables. As stated by Nadkarni and Shenoy (2001), Bayesian network is a kind cognitive (causal) map with probability tables for deterministic modeling or uncertainty-based modeling with the nodes, representing the concepts - random variables and the arcs, representing probabilistic relations and dependencies between the concepts-variables (Fenton et al., 2008). Finally, the last technique used in the proposed methodology is the Analytic Hierarchy Process (AHP) which is a multi-criteria decision making (MCDM) technique proposed by Saaty (1977, 1980). AHP uses reciprocal pairwise comparison matrix, as the cardinal transition between model variables by employing Right Eigenvector Method (EVM) for prioritization (Saaty, 1994).

For providing grounded constructs for our research we began with a literature study conducted on combinations of related topics. In order to find proper coverage the search identified the related concepts and subjects of our focus area. Initial step is the identifying potentially relevant variables of EA Components Variables to be used in the model for gathering the influence and relationship among them via Pairwise Comparison Matrix by expert elicitation and probability encoding methods. TOGAF 9.1 Standard was selected as the basis for Enterprise Architecture Framework in order to identify variables of ICT System Solution Selection model. TOGAF suggests main EA principles and also from related literature additional variables are added to the study list. TOGAF is the most prominent framework in EA frameworks. Additionally during the expert elicitation by survey the list of EA component variables were asked to the domain experts to evaluate for inclusion/exclusion. The rationale behind this is during the ICT system solution selection process the most effective or the most prior EA principles and EA components may be different from domain expert to domain expert according to their experience in their fields. So common EA Component variables by experts were selected as the model variables. With the EA Components the states of each variable are also included. The domain experts from 5 different companies were conducted for the survey from February to April 2018

This study is supposed to be a foundation for future works so EA Component Variables are limited for model testing and evaluation, more extended version of the model will be examined as a future work based on this study. Consequently 15 EA component variables were included for expert elicitation. The selected EA components are Business Continuity, Regulatory Compliance, Shared Resources-Common Use Applications, Protection of Data, Data Backup, Data Validation, Real-time Integration, Technology Independence - Control Technical Diversity, Simple Solutions - Ease of Use, Interoperability, Mobility, ICT Solution Selection, Flexibility, High Availability, Least Permissions.

\section{NUMERICAL EXAMPLE}

This section provides the procedure for building the Pairwise Comparison Matrix, deriving Causal Map, converting the Causal Map to Bayesian network for structure learning of a (discrete variable) network from domain expert. A Causal/Cognitive map indicates how domain expert performs and identify set of relationships between EA Component variables. In general, the relations represented in a Bayesian network are not necessarily to be causal (Sahin, Ulengin\&Ulengin 2005).

This step involves determining the interdependencies between EA component variables of interest via structured survey in order to reflect personal opinions of the domain experts' working in the field as preparatory background for getting insight and building causal map for EA components for ICT System Solution Selection. Due to foundational purpose of this study limited number of domain experts were selected to gain insights for validation and usefulness of model for future works. So 15 domain experts were invited to participate for "Quantitative Assessment Model Survey for ICT Solution Selection". These domain experts were ICT Team members from different sectors with different expertise levels, so in our future work expertise level based weighting mechanism will be added to the model. The survey as structured elicitation technique was designed with closed questions for the clarification purpose.

The structured survey was designed based on confirmatory approach to capture domain information as an expert elicitation technique. With the survey domain experts were asked with the following question structure: "How much more is required for EA Component Variable 1 preferred over EA Component Variable 2?" By considering this question, the domain experts were encouraged to identify associational relationships of EA Components in terms of their contribution to effective ICT solution selection and the probabilistic influential relationships among EA component variables defining the direction in order to build an adjacency matrix as positive relation with $\rightarrow$ sign, negative relation with $\leftarrow$ sign or no relation and signs show the direction of the link. Additionally the experts were commented on the influential relationship scale according to their insights on EA Component variables. This provides probability assessment from a scale. In order to properly characterize the response of the all domain experts all responses were combined according to frequency of each EA Component variables and resultant output were gathered consistently from each survey. After completion of survey from each respondent, Cronbach's Alpha test was conducted for reliability and validity of the survey answers. The result was over 0.7 that means acceptable reliability. The experts compared those variables in an adjacency matrix with the rows and the columns that list the EA component variables and they specified direction of the relation between each pair of EA components variables. Subsequently considering the output of adjacency matrix the Causal map has been produced. Next step is the derivation of causal map with the help of Netica Software. Initial causal map is transferred to Bayesian Network. Bayesian Network which is built from causal maps, is used to develop a proposed framework on scenario-based assessment of ICT System Solution in terms of EA and EE principles and Probabilistic 
Inference Process among set of EA Component variables. The quality of the model as a representation is assessed using AHP technique. It follows that our allowable consistency ratio should be not more than about 0.10 as calculated in our proposed model according to background data from our survey. Preliminary results demonstrated the practical applicability and efficiency of the proposed model.

\section{CONCLUSION}

With Digital age ICT investments are growing very fast and require considerable amount of funds available in Enterprise organizations. Consequently, it would be desirable to be able to decide ICT projects with proper EA strategy and principles in order to increase benefits from ICT projects. It is obvious that replacing one IT investment by another is not desirable decision for cost controlling target nearly all of Enterprise Organizations. It has already been highlighted in our study that uncertain information may cause deficiencies for the evaluation and comparison of ICT projects. In general, ICT projects selection is rather characterized by high uncertainty and risks. Due to lack a comprehensive decision mechanisms for ICT System Solution selection, the proposed model may support decision makers during this crucial task and standard framework based approach for business and technical needs of Enterprise organizations. Our research also conducted an empirical and quantitative evaluation of the EA and EE approach for the realization decision of ICT projects. Using data from a survey among domain expert from various sectors and companies can provide another perspective on maturity level of EA approach and a practical perspective on the theoretical EA framework.

In Further steps of this study, we will be testing the proposed model in a particular holding company application in order to see whether the results indicate that the proposed model provides satisfactory solutions. While this study provides an overview of the field of enterprise architecture and enterprise engineering, many aspect needs may be scanned for further refinement. Consequently case studies can be renewed according to updated model in terms of performance and efficiency effects.

\section{REFERENCES}

Dietz, J. L. G., Proper, E., Tribolet, J. (2009). Enterprise governance and enterprise engineering

Dietz, J. L., Hoogervorst, J. A. (2011). A critical investigation of TOGAF-based on the enterprise engineering theory and practice. In Enterprise Engineering Working Conference (pp. 76-90). Springer, Berlin, Heidelberg.

Domingos, P., Pazzani, M. (1997). On the optimality of the simple Bayesian classifier under zero- one loss. Machine Learning, $29,10330$.

Eden, C. (2004). Analyzing cognitive maps to help structure issues or problems. European Journal of OperationalResearch 159(3) 673-86.

Fenton, N., Neil, M., Marquez, D. (2008). Using Bayesian networks to predict software defects and reliability. Proceedings of the Institution of Mechanical Engineers, Part O: Journal of Risk and Reliability, 222(4), 701-712.

Greefhorst, D., Proper, E. (2011). The role of enterprise architecture. In Architecture Principle.

Harary, F. (1972). Graph theory. Addison-Wesley, Reading.

Harary, F., Norman, R., Cartwright, D. (1965). Structural models: an introduction to the theory of directed graphs. Wiley, New York.

Kosanke, K., Vernadat, F., Zelm, M. (1999). CIMOSA: Enterprise engineering and integration. Computers in Industry $40(2): 83-87$.

Nadkarni, S., Shenoy, P. P. (2001). A Bayesian network approach to making inferences in causal maps. European Journal of Operational Research 128 (2001) 479-498.

Robertson, S. E., Sparck Jones, K. (1976). Relevance weighting of search terms. J. American Society for Information Science, 27, 129-146.

Saaty, T. L. (1977). A scaling method for priorities in hierarchical structures. Journal of mathematical psychology, 15(3), $234-281$.

Saaty, L. (1980). The analytic hierarchy process. New York: McGraw-Hill.

Saaty, T. L. (1994). Fundamentals of decision making.

Sahin, S. O., Ulengin, F., Ulengin, B. (2005). A Bayesian causal map for inflation analysis: the case of Turkey. European Journal of Operational Research: 1268-84.

Sessions, R. (2007). A comparison of the top four enterprise-architecture methodologies. Houston: ObjectWatch Inc.

Yildiran, P., Kilic, H. S., Sennaroglu, B. (2018). Collaborative system approach for enterprise engineering and enterprise architecture: a literature review. In Enhancing Competitive Advantage with Dynamic Management and Engineering (pp. 138-216). IGI Global. 